\title{
How are medical groups identified as high- performing? The effect of different approaches to classification of performance
}

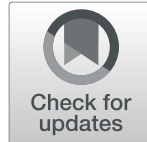

Sangeeta C. Ahluwalia ${ }^{1,2^{*}}$ (D, Cheryl L. Damberg ${ }^{1}$, Ann Haas $^{3}$ and Paul G. Shekelle $e^{1,4}$

\begin{abstract}
Background: Payers and policy makers across the international healthcare market are increasingly using publicly available summary measures to designate providers as "high-performing", but no consistently-applied approach exists to identifying high performers. This paper uses publicly available data to examine how different classification approaches influence which providers are designated as "high-performers".
\end{abstract}

Methods: We conducted a quantitative analysis of cross-sectional publicly-available performance data in the U.S. We used 2014 Minnesota Community Measurement data from 58 medical groups to classify performance across 4 domains: quality (two process measures of cancer screening and 2 composite measures of chronic disease management), total cost of care, access (a composite CAHPS measure), and patient experience (3 CAHPS measures). We classified medical groups based on performance using either relative thresholds or absolute values of performance on all included measures.

Results: Using relative thresholds, none of the 58 medical groups achieved performance in the top 25\% or $35 \%$ in all 4 performance domains. A relative threshold of $40 \%$ was needed before one group was classified as high-performing in all 4 domains. Using absolute threshold values, two medical groups were classified as high-performing across all 4 domains. In both approaches, designating "high performance" using fewer domains led to more groups designated as high-performers, though there was little to moderate concordance across identified "highperforming" groups.

Conclusions: Classification of medical groups as high performing is sensitive to the domains of performance included, the classification approach, and choice of threshold. With increasing focus on achieving high performance in healthcare delivery, the absence of a consistently-applied approach to identify high performers impedes efforts to reliably compare, select and reward high-performing providers.

Keywords: Performance measurement, Quality, Health system

\section{Background}

Improving the performance of healthcare providers (i.e., healthcare delivery systems, hospitals, and medical groups) is a principal health policy goal across international healthcare markets [1-5]. Payers and policy makers are applying a variety of levers to stimulate performance improvement by healthcare providers including public reporting of performance and recognition

\footnotetext{
*Correspondence: sahluwal@rand.org

'RAND Corporation, 1776 Main Street, Santa Monica, CA 91403, USA

${ }^{2}$ UCLA Fielding School of Public Health, Los Angeles, CA, USA

Full list of author information is available at the end of the article
}

(e.g., CMS Star ratings in the U.S. or the UK's National Health Service's star ratings and Quality Outcomes Framework and financial incentives to providers designated as "high performing" [1-3, 6-12]. Pay-forperformance programs are widely used in the U.S. by public and private insurers and increasingly used in Canada, Australia, U.K, and other European countries, and even in some countries in the developing world [5, 13-15]. Patients are encouraged to choose higher performing providers using publicly available scorecards that rate and classify provider performance.

(c) The Author(s). 2019 Open Access This article is distributed under the terms of the Creative Commons Attribution 4.0 International License (http://creativecommons.org/licenses/by/4.0/), which permits unrestricted use, distribution, and 
Designating a provider as "high-performing" requires an agreed-upon definition of high performance with standard decision rules and performance measures. Our recent systematic review of definitions of high performance found no consistent definition of what constitutes a high-performing provider or healthcare system [16]. Wide variation exists in how payers and researchers designate "high-performing" providers, applying different performance domains (e.g., quality, cost, access, patient experience) and types of measures (e.g., individual vs. composite measures) within those domains, and using varying thresholds. While situations may exist where focusing on one or two aspects of performance is important, designating providers as "high-performing" based on only certain performance domains (e.g., clinical quality) risks identifying providers as "high-performing" despite poor performance in other important domains (e.g., patient experience, cost). As consumers are being encouraged and even financially incentivized to obtain all or most of their care within a single health system or a medical group, it is reasonable for consumers to expect that the system or medical group they choose should be high performing across multiple dimensions of performance. Similarly, the use of individual versus composite measures has implications for how stakeholders perceive and understand highperformance [17]. Individual measures can characterize performance within single domains and identify specific processes for improvement within groups, while composite measures combine different aspects of performance and facilitate benchmarking across groups. While the merits of each of these approaches can be argued and improved upon, consumers, payers, and policy makers are typically limited to the domains and measures included in existing publicly available data for assessing provider performance and selecting or rewarding the "high-performers". It is important to understand - through the data currently available to stakeholders - the extent to which different applications of the definition and measurement of performance impact if and how groups are identified as high-performing.

In the current study, we sought to understand the real-life implications of using different performance domains and classification approaches on designating providers as high-performers. Specifically, we assessed existing, publicly-reported provider performance on four commonly-used performance domains identified in our prior review [16] - quality, cost, access, and patient experience - and applied two different classification approaches, relative and absolute performance thresholds, to test whether different approaches resulted in different providers being designated as high-performers.

\section{Methods}

\section{Data source}

We used publicly available performance data from the 2014 Minnesota Community Measurement (MNCM)
Health Care Quality Report (http://mncm.org/healthcare-quality-report/), which reports comparative medical group performance data. The MNCM report is used by providers, payers, employers, and policymakers to guide improvement and investment efforts and by consumers as guidance for selecting among providers [18]. The medical groups in MNCM data include any combination of primary, specialty, or multi-specialty provider organizations operating under the same tax identification number. Measures included in the MNCM data were chosen by a multi-stakeholder group in Minnesota to address gaps in performance and stimulate improvement efforts [18].

\section{Measures}

Drawing upon the Institute of Medicine's (IOM) conceptual framework for a twenty-first century health system that is safe, effective, patient-centered, timely, efficient, and equitable [19], we examined medical group performance across 4 of the 6 IOM performance domains, that were also identified in our prior review [16]: quality, total cost of care, access, and patient experience. There were no available measures in our dataset to assess performance in the IOM domains of safety and equity.

For each of the four performance domains, we applied an "all-or-none" approach to defining high-performance $[20,21]$, wherein a medical group had to be highperforming on all measures within a domain, thus requiring the medical group to report on all selected measures. To maximize the number of medical groups in our sample given this "all-or-none" approach, and to ensure we were including similar types of medical groups for comparison, we selected the subset of measures within each domain reported by the largest number of medical groups, and excluded specialized measures reported by only a small number of groups, e.g., primary C-section rates (See Appendix for a full list of all measures available in the MNCN data). The included measures address ambulatory care provided by both primary care physicians and specialists and reflect commonly targeted measures for ambulatory care improvement:

- Quality: We selected 4 measures to represent the quality domain: 1) colorectal cancer screening; 2) breast cancer screening; 3) optimal diabetes care composite; 4) optimal vascular care composite.

- Cost: The National Quality Forum-endorsed total cost of care measure, which represents the average cost of care per member per month for all patients within each medical group. The methodology [22].

- Access: A composite measure from the Consumer Assessment of Healthcare Providers and Systems Clinician and Group (CG-CAHPS) survey that assesses the availability of appointments, access to 
routine and urgent care, and information when needed over the past year.

- Patient Experience: We used 3 CG-CAHPS measures: courteous and helpful staff, how well providers communicate, and providers with the most positive rating.

While MNCM reports some measures at both the clinic and the medical group level, our analysis focused on the medical group as the unit of analysis because more measures of performance were reported at this level.

\section{Analytic sample}

Of the 240 medical groups in the MNCM database, 78 reported data on all four selected quality measures (i.e., optimal diabetes care, optimal vascular care, colorectal cancer screening and breast cancer screening). Of the 78 medical groups reporting all four quality measures, 67 (86\%) also reported cost data, and of these, 58 (74\%) also reported the selected CAHPS measures on access and patient experience. Our final analytic sample includes 58 general medical groups reporting all selected measures across the 4 domains.

We used an annually published list of the Top 25 medical groups in Minnesota by revenue to assess the generalizability of our sample. Of the Top 25 medical groups in Minnesota by revenue in 2015 [23], 16 provided a broad range of adult medical care services (e.g., primary care, multi-specialty), while 9 provided single specialty care only (e.g., dental, senior home care, pediatrics). Of the 16 groups providing adult medical care (i.e., the groups most relevant to our study), 14 (88\%) reported data across all 4 performance domains of interest and are included in our analytic sample. Thus, our sample includes almost all the largest general medical groups in Minnesota in terms of revenue; i.e., the medical groups likely to be where most Minnesotans receive their care.

Comparing included and non-included groups showed that about half of the 240 groups did not report a measure of interest. For example, 127 groups did not report the diabetes composite measure and 106 groups did not report the breast cancer screening measure. The primary reasons for not reporting were that the measure did not apply to the medical group's patient population (e.g., a pediatric group or an orthopedic group) or the sample size of patients was too small to generate a stable estimate. Included groups performed about $10 \%$ better than non-included groups on each reported quality measure (example: median vascular composite measure scores for included versus non-included groups was $62.3 \%$ vs $59.3 \%$ ) and there was a narrower distribution of scores within each measure among included groups. Included groups also were about $5 \%$ higher in cost.

\section{Analyses}

We pre-specified the conceptual methods for our analyses. We classified medical groups as high performing using two threshold approaches commonly used in practice to classify providers: 1) relative value thresholds, where groups are ranked by performance relative to each other (e.g., top 25\%, top 35\%) and 2) absolute value thresholds, where groups are ranked according to pre-set or objective standards (e.g. scores above 75\%, scores above 90\%). Both approaches have strengths and weaknesses [24, 25].

\section{Relative value threshold approach}

We first ordered each medical group according to its performance in each of the four domains, and for those domains with multiple measures, on each measure within domain. For example, using a top quartile relative value threshold, a medical group had to be in the top $25 \%$ of performance for each of the four measures comprising the quality domain to be classified as performing in the top quartile for quality. To be a high performer for the cost domain, a medical group would have to perform in the lowest quartile of average costs per member per month. We tested numerous relative threshold values: top $25 \%$, top $35 \%$, top $40 \%$, top $50 \%$.

\section{Absolute value threshold approach}

We identified absolute score thresholds for each performance measure. Initial attempts at using a stringent absolute score threshold such as $90 \%$ (the equivalent of an "A" grade) or $80 \%$ (a "B" grade) for all measures found no medical groups would be classified as high performing. Conversely, setting the absolute threshold low enough (e.g., 50\%) such that some groups would be classified as high performing on the most difficult to attain measure (the composite diabetes measure) meant that most groups were high performing on all other measures. Choosing a $50 \%$ absolute threshold for all domains would be tantamount to distinguishing medical groups based solely on their diabetes care and has limited face validity, as consumers and policymakers reasonably expect high performance to mean more than an "F" grade. We therefore set $66.6 \%$ as an initial absolute score threshold for the quality measures and $80 \%$ for the access and patient experience measures. From this initial threshold, we adjusted within each domain to avoid situations where almost all or no groups were high performing on any individual measure, resulting in the following absolute value thresholds:

- Quality - The absolute score threshold for diabetes care was set at $>0.50$, for vascular care and colorectal cancer screening at $>0.66$, and for breast cancer screening at $>0.75$.

- Patient Experience: The absolute score threshold for each of the 3 CAHPS metrics was set at $>0.80$.

- Access: The absolute score threshold for the CAHPS composite measure was set at $>0.60$. 
Using the lowest quartile of costs $(<\$ 420$ average monthly cost of care per patient) no groups would be classified as high performing (while being high performing in other domains); therefore, we selected the 50-percentile ( $\leq \$ 457$ average monthly cost of care per patient) as the absolute value threshold. This value represents the bottom half of all medical groups, and we judged that being able to deliver high performance on the domains of quality, patient experience and access using the above absolute value thresholds while keeping costs at or below the average had face validity as being "high performing".

When we assessed performance across multiple domains, we only assessed combinations of domains that included quality, as this is by far the most common domain included in existing operational multi-domain definitions of performance [16].

\section{Results}

Among the 58 medical groups, the median number of clinics in each group was three, and the median number of physicians was 34 .

\section{Relative threshold classification approach}

Based on a top 10, 25\%, or 35\% threshold approach, no medical groups were identified as high performing across all four performance domains. A single medical group was identified using a top $40 \%$ threshold and a second medical group was identified using a top 50\% threshold (Table 1).

Examining relative performance across combinations of three of the four performance domains, several medical groups were identified applying a top 35\% threshold; however, there was little concordance in which medical groups were classified as high performers across the different combinations of performance domains (Fig. 1). For example, CentraCare Health and Gundersen performed in the top 35\% for quality, access, and patient experience but not when measured across quality, access, and cost. Instead, a different set of groups - Affiliated, Health Partners and Stillwater - performed in the top 35\% of quality, access, and cost (Park Nicollet and Allina performed in the top 35\% for both combinations of the three domains). The difference in a medical group's classification as high performing when exchanging a single domain (e.g., patient experience and cost) could be slight and with little implications for practice, (e.g., one group had patient experience measures ranking 12th, 22nd, and 29th, and another group ranked 22rd in cost, but ranking 21st for all measures within a domain would have been sufficient to be designated within the top $35 \%$ of performance) or it could be large and have important practice implications (one group ranked in the bottom 25\% for patient experience, another group ranked 57th in cost, meaning it was the second-most-expensive group).
Table 1 Effect of different relative and absolute value classification methods on classification of medical groups as high performing

\begin{tabular}{ll}
\hline Threshold for high performing & $\begin{array}{l}\text { Number of groups classified } \\
\text { as high performing }\end{array}$ \\
\hline Relative value method & 0 \\
Top 10\% in all domains & 0 \\
Top 25\% in all domains & 0 \\
Top 35\% in all domains & 1 \\
Top 40\% in all domains & 2 \\
Top 50\% in all domains & \\
Absolute value method & 0 \\
Achieving $90 \%$ on all measures & 0 \\
Achieving $80 \%$ on all measures & 0 \\
Achieving 70\% on all measures & 3 \\
Achieving 60\% on all measures & 16 \\
Achieving 50\% on all measures &
\end{tabular}

More groups were classified as high performers at the top $35 \%$ threshold across various combinations of performance domains when only assessed on two of the four performance domains; however, concordance in the groups identified as higher performers across different combinations of performance domains was limited.

\section{Absolute value classification approach}

As expected, using uniform absolute value thresholds across all domains did not result in useful identification of highperforming groups. No groups were identified with thresholds of $90,80 \%$ or $70 \%$, only 3 groups were designated high performing at a threshold of $60 \%$, and a threshold of $50 \%$ is roughly equivalent to distinguishing between groups solely on the basis of the diabetes composite measure (Table 1).

Using variable absolute value thresholds for each of the metrics identified many more groups as high performers in the individual domains. For example, nearly $40 \%$ of the groups were identified as high performers in the domain of Patient Experience, and half of the groups were identified as high performers in the domains of Access and Cost. Two medical groups were identified as high performing across all four domains (Fig. 2). Similar to the relative value approach, more groups were classified as high performers with combinations of two performance domains rather than three or four domains of performance. For example, when assessing performance in quality and cost, eight medical groups were identified as high-performers, but only five of those medical groups continued to be classified as highperformers when adding the access domain.

There was only moderate concordance across combinations of performance domains in which groups were designated as high performing. For example, there were seven medical groups identified as high-performing for both quality and patient experience, but only three of those 


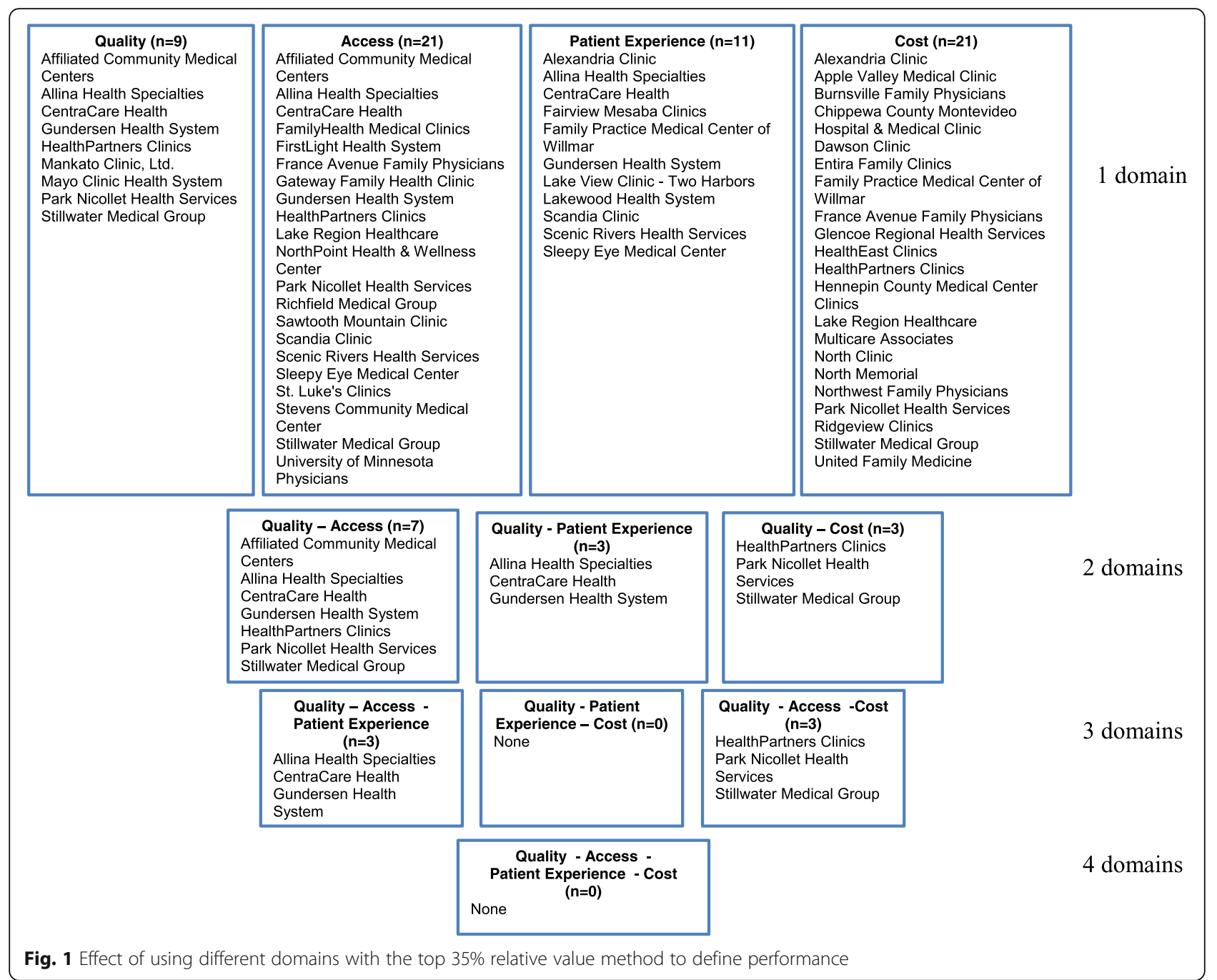

groups were also identified as high-performing for quality and cost. Five other medical groups met the threshold for high-performance in quality and cost, but only two of them met the criteria for high-performing in quality and access. As in the relative value approach, the reasons for the different groups being identified in one combination of domains but not another were in some cases small (one medical group had a score for access that was 58.5\%, where $60 \%$ was needed to be classified as high performing) and in some cases very large (one group had an average cost of $\$ 823$ when the threshold was $\$ 457$ ).

\section{Discussion}

The aim of our study was to understand how different definitional and measurement approaches to classifying performance affects which medical groups are identified as high-performing. We found the classification of medical groups as high-performing is highly sensitive to 1) the performance domains included and 2) the thresholds used within each of the domains to define performance as "high". Regardless of threshold approach used, very few Minnesota medical groups performed in the top $50 \%$ of the distribution when assessed across all measures and no groups performed in the top tertile of all four domains. Our study also finds fewer medical groups were identified as high-performing when more domains are used to evaluate performance.

We used publicly available data currently used by consumers and payers to understand the "real-life" implications of different classification approaches. As such, we are subject to the limitations of the data we used. For example, we were limited to the number and type of measures collected and reported within the MNCM dataset. As with most measurement schemes, the clinical measures represent a fraction of all care provided, although the clinical areas measured affect a substantial portion of patients and the measures of patient experience and cost of care encompass the entire medical group's patient population. MNCM does not assess performance in the domains of safety and equity; thus, we were only able to 


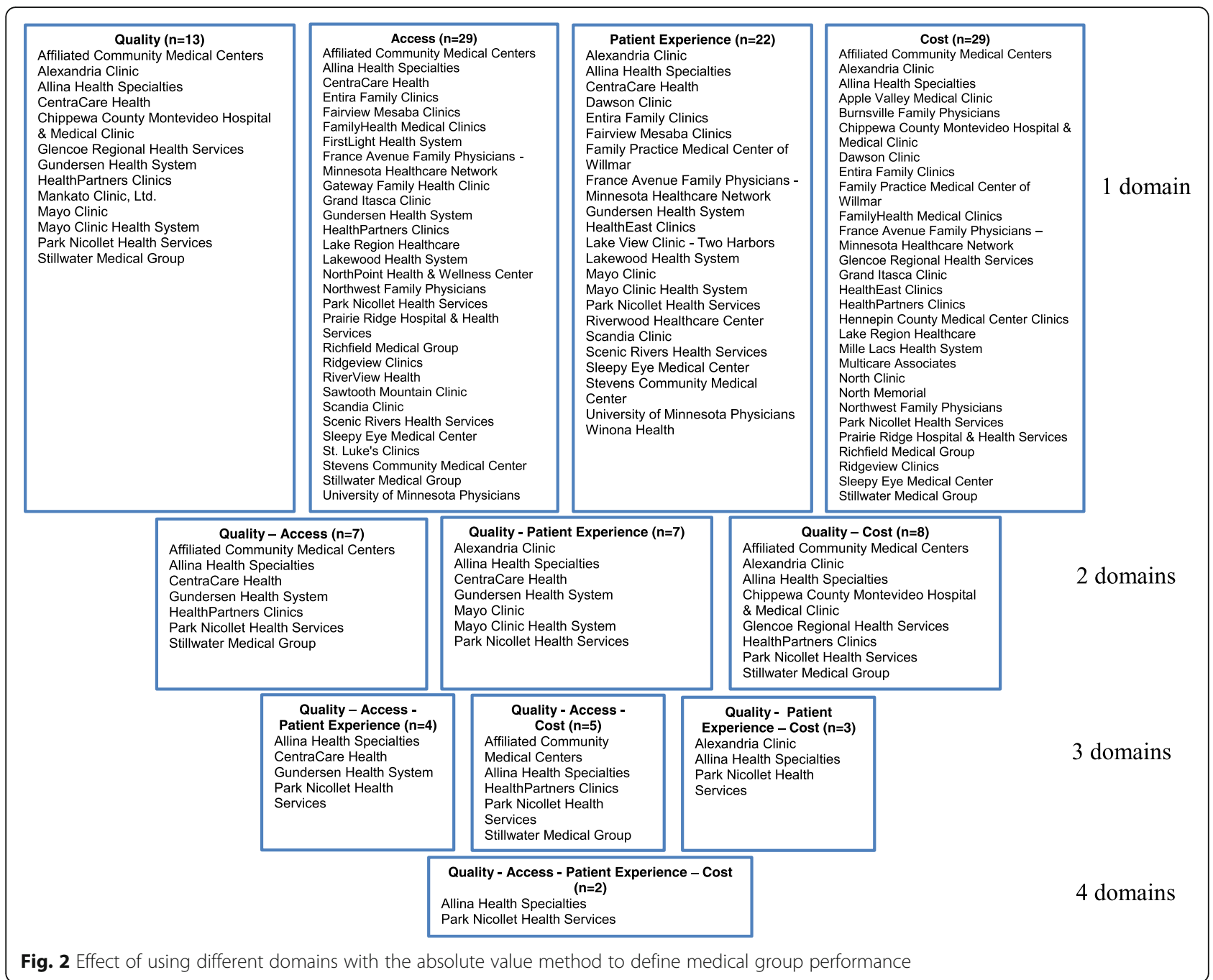

assess performance in the commonly-used domains of quality, cost, access, and patient experience.

Another limitation of the MNCM group-level performance data is the inability to examine the extent to which differences in case mix may influence performance, particularly for social risk factors, a concern that has been raised by a number of bodies [26-29]. To examine this would require person-level data which were not available. We note that the CAHPS measures and Total Cost of Care measures are adjusted for differences in the patients across groups, whereas the clinical measures, in keeping with National Committee for Quality Assurance (NCQA)-Healthcare Effectiveness Data and Information Set (HEDIS) measure specifications, are not. While not the aim of our study, future work might examine ways to improve performance measurement to account for differences in patient characteristics. Finally, while there may be some measurement error inherent in the different measures included in the MNCM dataset, the MNCM imposes denominator thresholds (e.g.,
NCQA-HEDIS minimum reporting thresholds) to ensure that the estimates of performance are reliable enough to facilitate the ability to discriminate provider performance.

The absence of a consistent approach to measuring and classifying "high-performance" has practical implications beyond our study; for example, the CMS Star Ratings program designates high performance using a clustering algorithm based on relative thresholds while the Integrated Healthcare Association (IHA) uses an absolute threshold of $50 \%$ to designate high performance [30]. The same medical groups selected for high performance in one program may not similarly be designated in another program. This creates potential confusion for consumers and sends conflicting messages to the providers being evaluated about what constitutes high performance.

A key measurement challenge facing program sponsors when benchmarking performance is how to set meaningful thresholds for classifying high-performing providers. Absolute value thresholds [24] have the distinct benefit of holding providers to an external and objective standard 
(similar to the "A", "B", "C" grades given in school) and allowing providers to target investments in improvements with specific aims. Our work showed that setting a high standard such as a score of $90 \%$ (an "A" grade) identified no groups as high-performing and would leave patients and payers with no high-performing provider options to choose from and reward. Applying a lower standard such as 50\% (an "F" grade) identified most groups as highperforming on all performance measures except one, leaving patients and payers with too many indistinguishable options. In contrast, relative thresholds provide strong improvement incentives because there is no absolute level at which reward and a designation of high performer is guaranteed. Relative thresholds also allow patients to compare and select providers on the basis of average performance which may be more useful to consumers. However, relative thresholds risk rewarding poor performance when the distribution of performance is low. Whether consumers should be told there are no high-performing providers to choose from (in the case of an absolute value approach where no one earns an A grade), or that they can choose from among the "top of the pack" providers whose actual performance might be low is a dilemma payers and policymakers continue to struggle with.

Our study has some methodological limitations. We used data from a single state which may limit the generalizability of our results; however, the variation in performance on individual measures in Minnesota is consistent with variations in and levels of performance seen in data from other studies [31-33]. Although not all Minnesota medical groups were represented in our study due to the "all-or-none" approach to selecting groups for inclusion, we included nearly all major multi-specialty medical groups operating within Minnesota, supporting the representativeness of our sample and increasing the generalizability of our findings. We were limited to commonly measured domains and measures of performance; however, increasing the number of measures within each domain or the number of domains used to define performance would likely only increase the variability in how groups are classified as high-performing. Lastly, to the degree chance plays a role in determining a provider's performance score on any particular measure in the MNCM dataset, that contribution of chance is incorporated into our results.

\section{Conclusion}

As health care markets increasingly embrace value-based purchasing to stimulate improvement in quality and costs, a major impediment to achieving these goals is the differing approaches used to classify providers as high-performing. Our results show that differences in how "high performing" is defined may result in completely different providers being so designated, even when using the same performance measures, creating confusion for providers in how to respond. To enable common identification of high

\section{Appendix}

Table 2 Measures publicly-available within the MNCM dataset

Performance Domain Measure

Quality

Breast Cancer Screening

Quality

Colorectal Cancer Screening

Quality

Diabetes

Quality

Vascular Disease

Patient Experience

Patient Experience

Patient Experience / Courteous and helpful office staff

Patient Experience / How well

Patient Experience

Cost

Access providers communicate

Patient Experience / Providers with a most positive rating

Total Cost: Overall

Quality (measures not selected ${ }^{\mathrm{a}}$ )

Patient Experience / Getting care when needed

Adolescent Immunization

Asthma - Adults

Asthma - Children

Bronchitis

COPD

Cervical Cancer Screening

Childhood Immunization

Chlamydia Screening

Colds

Depression - PHQ-9 Follow-up at 12 Months

Depression - Use of the PHQ-9

Depression Remission at 12 Months

Depression Remission at Six Months

Depression Response at 12 Months

Depression Response at Six Months

Depression: Follow Up (6 Months)

High Blood Pressure

Maternity - Cesarean Deliveries

Mental Health Screening: Teens

Overweight Counseling: Children

Sore Throats

Spinal Surgery: Lumbar Discectomy/ Laminotomy w/Pre-Op \& Post-Op OD

Spinal Surgery: Lumbar Fusion w/ Pre-Op \& Post-Op ODI

Cost (Measures not selected ${ }^{\mathrm{a}}$ ) Total Cost: Adults

Total Cost: Pediatrics

Total Knee Replacement: Assessing

Symptoms Before and After Surgery

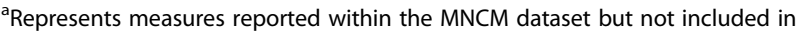
our study because 1) they were not reported by a majority of medical groups and/or 2) they are specialized measures only applicable to small subgroups 
performers for use in provider payment and recognition and provider selection by consumers, an agreed-upon standard definition of high performance and approach to measurement is needed.

\section{Abbreviations \\ CAHPS: Consumer Assessment of Healthcare Providers and Systems; CG- CAHPS: Clinician Group Consumer Assessment of Healthcare Providers and Systems; CMS: Centers for Medicare and Medicaid Services; HEDIS: Healthcare Effectiveness Data and Information Set; IHA: Integrated Healthcare Association; IOM: Institute of Medicine; MNCM: Minnesota Community Measurement; NCQA: National Committee on Quality Assurance}

\section{Acknowledgements}

Not applicable

\section{Authors' contributions}

CLD, AH, and PGS made substantial contributions to conception and design and acquisition of data. All authors (SCA, CLD, AH, PGS) made substantial contributions to analysis and interpretation of data; were involved in drafting the manuscript or revising it critically for important intellectual content; and have given final approval of the version to be published. Each author participated sufficiently in the work to take public responsibility for appropriate portions of the content and have agreed to be accountable for all aspects of the work in ensuring that questions related to the accuracy or integrity of any part of the work are appropriately investigated and resolved.

\section{Funding}

This research was funded by the Agency for Healthcare Research and Quality (1U19HS024067-01). The funder had no involvement in study design; in the collection, analysis and interpretation of data; in drafting the manuscript; or in the decision to submit the article for publication.

\section{Availability of data and materials}

We used publicly available performance data from the 2014 Minnesota Community Measurement (MNCM) Health Care Quality Report (http:// mncm.org/health-care-quality-report/).

\section{Ethics approval and consent to participate}

Not applicable

\section{Consent for publication}

Not applicable

\section{Competing interests}

The authors declare that they have no competing interests.

\section{Author details}

${ }^{1}$ RAND Corporation, 1776 Main Street, Santa Monica, CA 91403, USA. ${ }^{2}$ UCLA Fielding School of Public Health, Los Angeles, CA, USA. ${ }^{3}$ RAND Corporation, Pittsburgh, PA, USA. ${ }^{4}$ VA West Los Angeles Medical Center, Los Angeles, CA, USA.

Received: 1 October 2018 Accepted: 23 June 2019

Published online: 18 July 2019

\section{References}

1. Ginsburg PB. Achieving health care cost containment through provider payment reform that engages patients and providers. Health Aff (Millwood). 2013;32(5):929-34

2. Delisle DR. Big things come in bundled packages: implications of bundled payment systems in health care reimbursement reform. Am J Med Qual. 2013:28(4):339-44

3. Conrad DA, Grembowski D, Hernandez SE, Lau B, Marcus-Smith M. Emerging lessons from regional and state innovation in value-based payment reform: balancing collaboration and disruptive innovation. Milbank Q. 2014:92(3):568-623.

4. Quality Outcomes Framework (QOF). https://digital.nhs.uk/data-andinformation/data-tools-and-services/data-services/general-practice-datahub/quality-outcomes-framework-qof. Accessed 28 June 2019.
5. Eijkenaar F. Pay for performance in health care: an international overview of initiatives. Med Care Res Rev. 2012:69(3):251-76.

6. Layton TJ, Ryan AM. Higher incentive payments in Medicare Advantage's pay-for-performance program did not improve quality but did increase plan offerings. Health Serv Res. 2015;50(6):1810-28.

7. Ryan A, Sutton M, Doran T. Does winning a pay-for-performance bonus improve subsequent quality performance? Evidence from the hospital quality incentive demonstration. Health Serv Res. 2014;49(2):568-87.

8. DeLancey JO, Softcheck J, Chung JW, Barnard C, Dahlke AR, Bilimoria KY. Associations between hospital characteristics, measure reporting, and the centers for Medicare \& Medicaid Services Overall Hospital Quality Star Ratings. JAMA. 2017;317(19):2015-7.

9. Trzeciak S, Gaughan JP, Bosire J, Mazzarelli AJ. Association between Medicare summary star ratings for patient experience and clinical outcomes in US hospitals. J Patient Exp. 2016;3(1):6-9.

10. Mannion R, Davies HT, Marshall MN. Cultural characteristics of "high" and "low" performing hospitals. J Health Organ Manag. 2005;19(6):431-9.

11. Groene O, Skau JK, Frolich A. An international review of projects on hospital performance assessment. Int J Qual Health Care. 2008;20(3): 162-71.

12. Taylor N, Clay-Williams R, Hogden E, Braithwaite J, Groene O. High performing hospitals: a qualitative systematic review of associated factors and practical strategies for improvement. BMC Health Serv Res. 2015;15:244.

13. Gillam SJ, Siriwardena AN, Steel N. Pay-for-performance in the United Kingdom: impact of the quality and outcomes framework: a systematic review. Ann Fam Med. 2012;10(5):461-8.

14. Aghaei Hashjin A, Kringos DS, Manoochehri J, Aryankhesal A, Klazinga NS. Development and impact of the Iranian hospital performance measurement program. BMC Health Serv Res. 2014;14:448.

15. Braithwaite J, Matsuyama $Y$, Mannion R, Johnson J. Healthcare reform, quality and safety: perspectives, participants, partnerships and prospects in 30 countries. Farnham, Surrey: Ashgate; 2015.

16. Ahluwalia SC, Damberg DL, Silverman M, Motala A, Shekelle PG. What defines a high-performing health care delivery system: a systematic review. Jt Comm J Qual Patient Saf. 2017;43(9):450-9.

17. Shwartz M, Restuccia JD, Rosen AK. Composite measures of health care provider performance: a description of approaches. Milbank Q. 2015;93(4): $788-825$.

18. MN Community Measurement: 2018 Minnesota Health Care Quality Report. February 2019. Available: https://mncm.org/reports-and-websites/reportsand-data/health-care-quality-report/. Accessed 28 June 2019.

19. Institute of Medicine: crossing the quality chasm: a new health system for the 21st century. Washington, DC; 2001

20. Nolan T, Berwick DM. All-or-none measurement raises the bar on performance. JAMA. 2006:295(10):1168-70.

21. Reeves D, Campbell SM, Adams J, Shekelle PG, Kontopantelis E, Roland MO. Combining multiple indicators of clinical quality: an evaluation of different analytic approaches. Med Care. 2007;45(6):489-96.

22. Total Cost of Care (TCOC) and Total Resource Use: A measurement approach to achieve the Triple Aim. https://www.healthpartners.com/ucm/groups/ public/@hp/@public/documents/documents/dev_057649.pdf. Accessed 28 June 2019.

23. The List: Health Care Providers. Ranked by Revenue. https://www.bizjournals. com/twincities/subscriber-only/2015/11/13/health-care-providers.html. Accessed 28 June 2019

24. Dudley AR, M.B. R: Pay for Performance: A Decision Guide for Purchasers. Contract No./Assignment No. 290-01-0001/298. Prepared for the Agency for Healthcare Research and Quality. 2006.

25. Institute of Medicine Board on Health Care Services. Committee on redesigning health insurance performance measures, payment, and performance improvement programs: rewarding provider performance. aligning incentives in Medicare incentives in Medicare (pathways to quality health care series). Washington: National Academies Press; 2007.

26. National Quality Forum: Evaluation of the NQF Trial Period for Risk Adjustment for Social Risk Factors. Washington, DC: NQF; 2017

27. National Quality Forum: risk adjustment for socioeconomic status or other Sociodemographic factors. Washington, DC: NQF; 2014

28. United States Department of Health and Human Services, Office of the Assistant Secretary for planning and evaluation: report to congress: social risk factors and performance under Medicare's value-based purchasing programs. Washington, DC; 2016 
29. National Academies of sciences, engineering, and medicine: accounting for social risk factors in Medicare payment: identifying social risk factors. Washington, DC: The National Academies Press; 2016.

30. Integrated Healthcare Assocaiation (IHA) Awards [https://www.iha.org/ourwork/accountability/value-based-p4p/awards]. Accessed 28 June 2019

31. Agency for Healthcare Research and Quality: CAHPS Database.

32. Chan PS, Oetgen WJ, Buchanan D, Mitchell K, Fiocchi FF, Tang F, Jones PG, Breeding T, Thrutchley D, Rumsfeld JS, et al. Cardiac performance measure compliance in outpatients: the American College of Cardiology and National Cardiovascular Data Registry's PINNACLE (practice innovation and clinical excellence) program. J Am Coll Cardiol. 2010;56(1):8-14.

33. Rossi MC, Nicolucci A, Arcangeli A, Cimino A, De Bigontina G, Giorda C, Meloncelli I, Pellegrini F, Valentini U, Vespasiani G, et al. Baseline quality-ofcare data from a quality-improvement program implemented by a network of diabetes outpatient clinics. Diabetes Care. 2008;31(11):2166-8.

\section{Publisher's Note}

Springer Nature remains neutral with regard to jurisdictional claims in published maps and institutional affiliations.

Ready to submit your research? Choose BMC and benefit from:

- fast, convenient online submission

- thorough peer review by experienced researchers in your field

- rapid publication on acceptance

- support for research data, including large and complex data types

- gold Open Access which fosters wider collaboration and increased citations

- maximum visibility for your research: over $100 \mathrm{M}$ website views per year

At BMC, research is always in progress.

Learn more biomedcentral.com/submissions 